\title{
Pulsed ultrasounds accelerate healing of rib fractures in an experimental animal model: An effective new thoracic therapy?
}

\author{
Norberto Santana-Rodríguez, $\mathrm{MD}, \mathrm{PhD},{ }^{\mathrm{a}, \mathrm{b}}$ Bernardino Clavo, $\mathrm{MD}, \mathrm{PhD},{ }^{\mathrm{a}, \mathrm{c}}$ Leandro Fernández-Pérez, $\mathrm{MD}$, \\ $\mathrm{PhD},{ }^{\mathrm{d}}$ José C. Rivero, MD, ${ }^{\mathrm{e}}$ María M. Travieso, MD ${ }^{\mathrm{f}}$ María D. Fiuza, MD, PhD, ${ }^{\mathrm{a}}$ Jesús Villar, MD, PhD, ${ }^{\mathrm{g}}$ \\ José M. García-Castellano, MD, PhD, ${ }^{\text {a,h }}$ Octavio Hernández-Pérez, BSc, PhD, and Antonio Déniz, MD, PhD
}

Objectives: Rib fractures are a frequent traumatic injury associated with a relatively high morbidity. Currently, the treatment of rib fractures is symptomatic. Since it has been reported that pulsed ultrasounds accelerates repair of limb fractures, we hypothesized that the application of pulsed ultrasounds will modify the course of healing in an animal model of rib fracture.

Methods: We studied 136 male Sprague-Dawley rats. Animals were randomly assigned to different groups of doses (none, 50,100 , and $250 \mathrm{~mW} / \mathrm{cm}^{2}$ of intensity for 3 minutes per day) and durations $(2,10,20$, and 28 days) of treatment with pulsed ultrasounds. In every subgroup, we analyzed radiologic and histologic changes in the bone callus. In addition, we examined changes in gene expression of relevant genes involved in wound repair in both control and treated animals.

Results: Histologic and radiologic consolidation was significantly increased by pulsed ultrasound treatment when applied for more than 10 days. The application of $50 \mathrm{~mW} / \mathrm{cm}^{2}$ was the most effective dose. Only the 100 and $250 \mathrm{~mW} / \mathrm{cm}^{2}$ doses were able to significantly increase messenger RNA expression of insulin-like growth factor 1, suppressor of cytokine signaling-2 and -3 , and vascular endothelial growth factor and decrease monocyte chemoattractant protein-1 and collagen type II-alpha 1.

Conclusions: Our findings indicate that pulsed ultrasound accelerates the consolidation of rib fractures. This study is the first to show that pulsed ultrasound promotes the healing of rib fractures. From a translational point of view, this easy, cheap technique could serve as an effective new therapeutic modality in patients with rib fractures. (J Thorac Cardiovasc Surg 2011;141:1253-8)

Trauma is the leading cause of death in patients younger than 40 years of age. ${ }^{1} \mathrm{Rib}$ fractures (RFs) are reported in $4 \%$ to $12 \%$ of trauma admissions, ${ }^{2}$ occur commonly in closed chest

From the Experimental Surgery Unit, ${ }^{\text {a }}$ Research Unit, Hospital Universitario Dr. Negrín, Las Palmas de Gran Canaria, Spain; Department of Respiratory Care, ${ }^{\mathrm{b}}$ Hospital San Roque, Las Palmas de Gran Canaria, Spain; Department of Radiation Oncology, ${ }^{\mathrm{c}}$ Hospital Universitario Dr. Negrín, Las Palmas de Gran Canaria, Spain; Department of Clinical Sciences, ${ }^{\mathrm{d}}$ Unit of Pharmacology, Molecular and Translational Endocrinology Group University of Las Palmas de Gran Canaria, Las Palmas

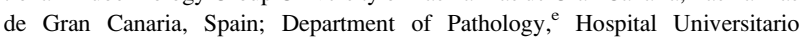
Dr. Negrín, Las Palmas de Gran Canaria, Spain; Department of Radiology, ${ }^{\mathrm{f}}$ Hospital San Roque, Las Palmas de Gran Canaria, Spain; CIBER de Enfermedades Respiratorias, ${ }^{\mathrm{g}}$ Instituto de Salud Carlos III, Madrid, Spain; Department of Orthopedic, ${ }^{\mathrm{h}}$ Complejo Hospitalario Materno-Insular, Las Palmas de Gran Canaria, Spain; and Department of Rehabilitation, ${ }^{\mathrm{i}}$ Hospital Universitario Dr. Negrín, Las Palmas de Gran Canaria, Spain.

Supported in part by a grant from the College of Physicians, Las Palmas de Gran Canaria, Spain. Norberto Santana (INT10/172) and Bernardino Clavo (INT10/030) are supported by the Instituto de Salud Carlos III, Madrid, Spain. Leandro Fernández-Pérez was supported by Spanish Ministry of Education and Science (SAF2006-07824).

Disclosures: Authors have nothing to disclose with regard to commercial support.

Received for publication March 23, 2010; revisions received May 18, 2010; accepted for publication June 20, 2010; available ahead of print Aug 16, 2010.

Address for reprints: Norberto Santana-Rodríguez, MD, PhD, Research Unit, Experimental Surgery Unit, Hospital Universitario Dr. Negrín, Barranco de la Ballena, s/n, 35010 Las Palmas de Gran Canaria, Canary Islands, Spain (E-mail: norbesr@ terra.es).

$0022-5223 / \$ 36.00$

Copyright (c) 2011 by The American Association for Thoracic Surgery

doi:10.1016/j.jtcvs.2010.06.028 trauma, and can cause pleuritic chest pain, pulmonary and/or extrapulmonary visceral injuries, and infectious complications. $^{1,2}$ In elderly persons, RFs are associated with a relatively high morbidity and mortality, which may reach up to $20 \%$ in case of severe pulmonary complications. ${ }^{3}$ The number of broken ribs is directly related to bad outcome owing to extrapulmonary causes. ${ }^{4}$ Currently, the management of $\mathrm{RF}$ is symptomatic, based on pain control, ${ }^{5}$ respiratory physiotherapy, and specific treatment of associated complications. Despite appropriate treatment of RF, functional disability often develops and can last several months, resulting in high hospital, pharmaceutical, labor, and social costs. ${ }^{6}$ The physiologic response to a bone fracture is the spontaneous initiation of a sequence of events including inflammation followed by a soft-callus formation, hard-callus formation, and bone repair and remodeling. ${ }^{7}$ This process involves the gene activation and synthesis of multiple growth factors that are expressed in a defined time sequence and, in some ways, it could be considered a recapitulation of endochondral bone development. ${ }^{8}$

Ultrasounds, widely used for diagnostic and therapeutic purposes, are a major therapeutic tool in physical medicine. Pulsed ultrasound (PUS) is a form of noninvasive mechanical energy that can be transmitted through the skin as a sound wave of high pressure often exceeding $20 \mathrm{MHz}$. Several experimental and clinical studies ${ }^{9,10}$ have shown that PUS with a 3-MHz frequency and intensity of $30 \mathrm{~mW} / \mathrm{cm}^{2}$ accelerates the 


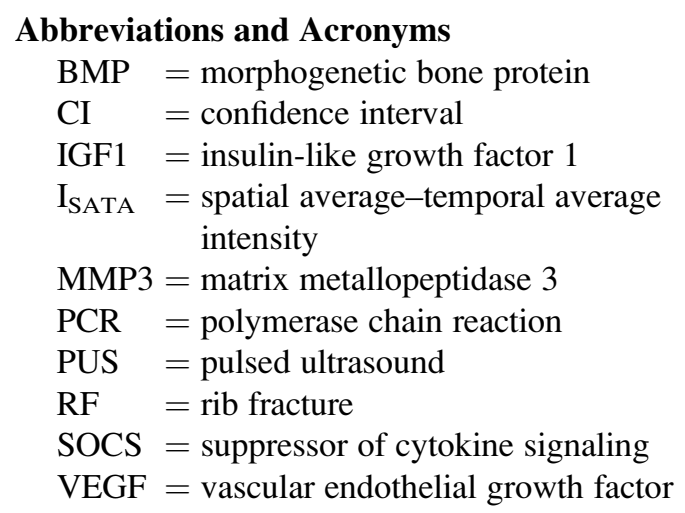

consolidation of recent limb fractures, prevents complications of fractures treated orthopedically, and accelerates the patient's return to his or her usual activities, thereby reducing costs and improving quality of life. The effect of PUS on fracture consolidation is dose- and time-dependent. ${ }^{11}$

To date, little is known of the molecular mechanisms underlying the effects of PUS or whether these effects can be extrapolated to other anatomic territories. This study represents the first evaluation of the effects of PUS on RF, in an in vivo animal model.

\section{MATERIAL AND METHODS}

This study was approved by the Institutional Review Board and Animal Care Committee at the Hospital Universitario Dr. Negrin Las Palmas de Gran Canarias, Spain.

\section{Study Design}

A total of 136 male Sprague-Dawley rats weighing between 300 and 400 $\mathrm{g}$ were used under optimum stabling conditions and randomly assigned for histologic and radiologic studies $(\mathrm{n}=88$, phase $\mathrm{I})$ and for genomic studies ( $\mathrm{n}=48$, phase II). For phase I, animals were randomly distributed in 4 groups: a control group $(n=40)$ and 3 other groups receiving PUS at a doses of $50(\mathrm{n}=16), 100(\mathrm{n}=16)$, and $250 \mathrm{~mW} / \mathrm{cm}^{2}(\mathrm{n}=16)$ of spatial averagetemporal average intensity $\left(\mathrm{I}_{\mathrm{SATA}}\right)$. Then, each group was divided into a function of time in $2,10,20$, and 28 days for controls ( $\mathrm{n}=10$ by subgroup) and for each group of PUS ( $\mathrm{n}=4$ by subgroups). For phase II, animals were randomly distributed in control $(\mathrm{n}=12), 50(\mathrm{n}=12), 100(\mathrm{n}=12)$, and 250 $\mathrm{mW} / \mathrm{cm}^{2} \mathrm{I}_{\text {SATA }}(\mathrm{n}=12)$, and each group was subdivided in 2 subgroups: 10 and 28 days ( $\mathrm{n}=6$ in each subgroup).

PUS treatment. PUS was applied through a Sonopuls 490 sonograph (Enraf-Nonius BV, Rotterdam, The Netherlands). We used a head of 3 $\mathrm{MHz}$, an area of effective radiation of $0.5 \mathrm{~cm}^{2}$, a beam nonuniformity ratio of 6 (which represents the ratio of the highest intensity in the field to the average intensity), and an $\mathrm{I}_{\mathrm{SATA}}$ of $0.5 \mathrm{~W} / \mathrm{cm}^{2}$ (which represents the maximum value occurring in time of the spatially averaged intensity), using pulses of 1 ms every $9 \mathrm{~ms}$ of pause when applied to $10 \%\left(50 \mathrm{~mW} / \mathrm{cm}^{2} \mathrm{I}_{\text {SATA }}\right)$ or $2-\mathrm{ms}$ pulse every $8 \mathrm{~ms}$ of pause when applied to $20 \%\left(100 \mathrm{~mW} / \mathrm{cm}^{2} \mathrm{I}_{\text {SATA }}\right)$ and 5 ms pulse with $5 \mathrm{~ms}$ of pause when applied to $50 \%\left(250 \mathrm{~mW} / \mathrm{cm}^{2} \mathrm{I}_{\mathrm{SATA}}\right)$. $\mathrm{I}_{\mathrm{SATA}}$, the spatial average-temporal average intensity, represents the total power in the beam divided by the beam area and averaged over the pulse repetition period. When evaluated at the transducer face, the head slipped in an area of skin of about $1.5 \mathrm{~cm}^{2}$. The total time of application by sitting on each animal was 3 minutes per day in a single session of therapy.
Experimental model. Animals were anesthetized by subcutaneous injection of $0.25 \mathrm{mg} / \mathrm{kg}$ of medetomidine and an intraperitoneal injection of $50 \mathrm{mg} / \mathrm{kg}$ of ketamine, intubated with an orotracheal tube, and mechanically ventilated (Servo 900; Siemens AG, Munich, Germany). A left thoracotomy was performed and a fragment of $0.5 \mathrm{~cm}$ of 2 adjacent ribs was removed. After all surgical procedures, animals received a subcutaneous injection of $0.25 \mathrm{mg} / \mathrm{kg}$ of atipamezole. All animals were treated with 0.025 $\mathrm{mg} / \mathrm{kg}$ per 12 hours subcutaneously of buprenorphine during the following 72 hours. After 24 hours of surgery, the treated animals received PUS daily until being put to death. A new thoracotomy was performed at 2,10,20, and 28 days. The bone callus was isolated and included in formaldehyde $1 \%$ for histologic study or was immediately snap-frozen in liquid nitrogen and stored at $-80^{\circ} \mathrm{C}$ for genomic studies. Animals were humanely killed in a chamber of carbon dioxide.

Histologic and radiologic evaluations. The bone callus specimens were serially sliced and processed in the usual manner, embedded in paraffin, then cut at $10-\mu \mathrm{m}$ thickness, stained with hematoxylin and eosin, and examined under a light microscope. A pathologist (J.C.R.) blinded to the experimental history of the bone specimens, performed the pathologic studies. Random sections of the callus from each animal were examined with particular reference to the presence and distribution of inflammatory infiltrate and extracellular matrix mineralization and classified in 3 categories: inflammatory, repair, and remodeling.

We performed a chest radiograph on all animals (Siemens Mobilett II; Siemens, Erlangen, Germany) at $55 \mathrm{KV}$ and $3.2 \mathrm{mAs}$ immediately after the operation and just before animal death. Radiologic images were evaluated by a radiologist (M.M.T.) blinded to the experimental history of the specimens. Images were classified in 3 categories: lack of callus, callus emerging, and callus formed.

Analysis of gene expression by quantitative real-time polymerase chain reaction (PCR). Total RNA was isolated from bone callus with TRIzol reagent (Invitrogen, Carlsbad, Calif) following manufacturer's instructions. For the purpose of this study, we examined the expression of several genes involved in bone repair and remodeling, such as morphogenetic bone protein-2 (BMP), collagen type II-alpha 1, insulin-like growth factor $1(I G F 1)$, monocyte chemoattractant protein-1, matrix metallopeptidase 3, transforming growth factor beta, vascular endothelial growth factor $(V E G F)$, polymerase II polypeptide $\mathrm{A}$, and for the first time, suppressor of cytokine signaling (SOCS)-2 and SOCS3. The cDNA synthesis was achieved by diluting $2 \mu \mathrm{g}$ of RNA in a final volume of 20 $\mu 1$ with random primers, deoxyribonucleotides, and Moloney murine leukemia virus reverse transcriptase (Promega Corporation, Madison, Wis) for 1 hour at $37^{\circ} \mathrm{C}$. Primers were designed using Primer 3 software. ${ }^{12}$ Real time PCR reactions were performed using LightCycler-FastStart DNA Master SYBR Green I (Roche Applied Science, Mannheim, Germany). Serially diluted samples containing pooled complementary DNAs from each animal were used for the standard curves. Achievement of a single PCR product was confirmed by agarose gel electophoresis.

\section{Statistical Analysis}

Data are expressed as group mean $\pm \mathrm{SD}$ or median, as appropriate. Comparisons among the categorical variables were assessed with the Fisher's exact test and $\chi^{2}$. Odds ratio with $95 \%$ of confidence interval (CI) were determined. The differences in the gene expression activity were tested with the Mann-Whitney $U$ test. Data management and statistical analysis were performed using the statistical package SPSS 14.0 (SPSS, Inc, Chicago, Ill).

\section{RESULTS}

\section{Postoperative Outcome}

Two animals from the control group and 1 animal from the $50 \mathrm{~mW} / \mathrm{cm}^{2}$ group (phase I) died during the first 24 hours after the operation owing to unexpected surgical complications 

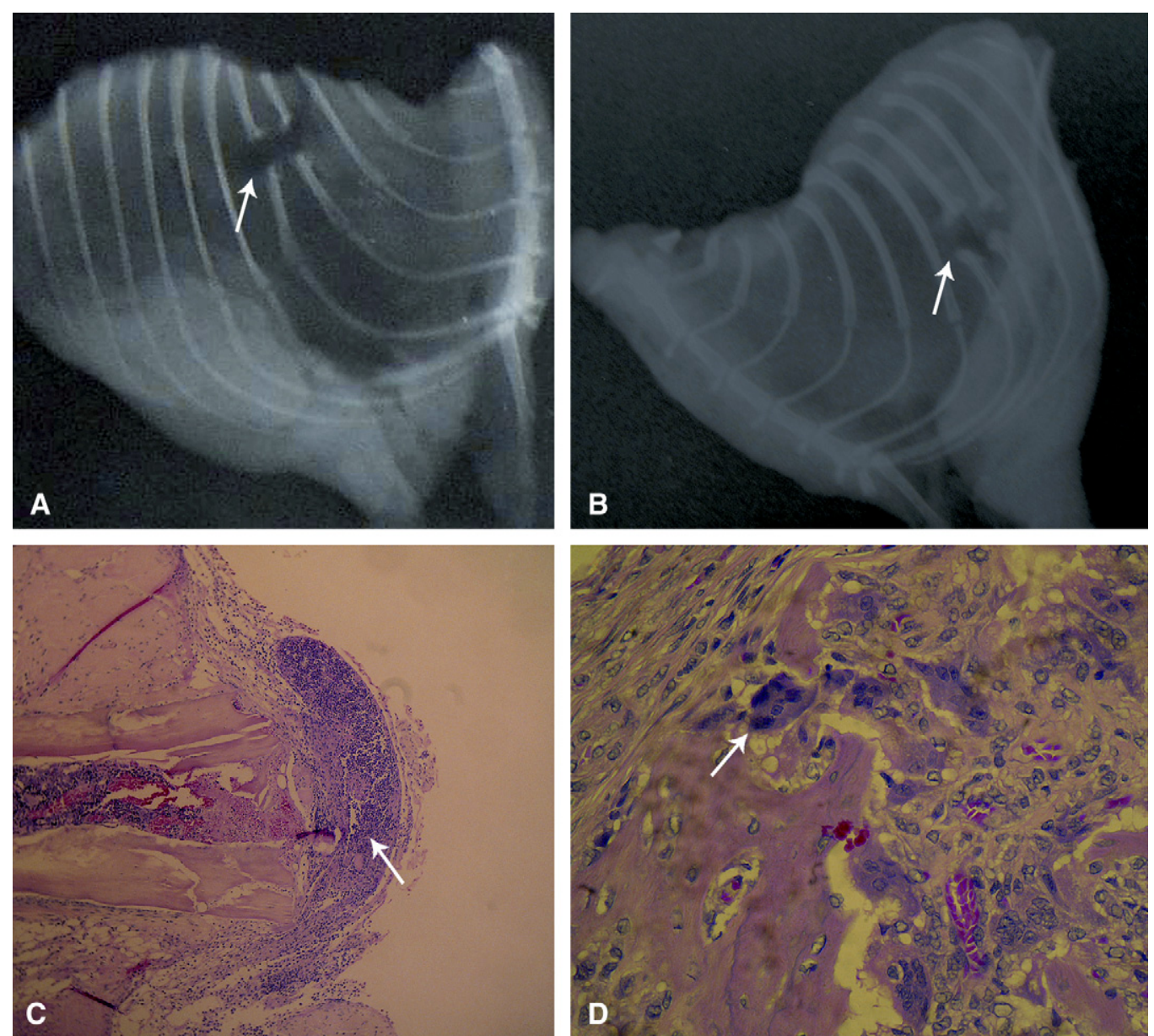

FIGURE 1. Radiologic and histologic findings after pulsed ultrasound treatment of rib fractures. A, No radiologic callus (arrow) was observed in 1 representative control animal at 28 days of treatment. B, Radiologic bone callus (arrow) and periosteal reaction in 1 representative animal treated with $50 \mathrm{~mW} / \mathrm{cm}^{2}$ at 28 days. C, Inflammatory infiltrate (arrow) and hemorrhage in 1 representative control animal at 28 days (original magnification $10 \times$ ). D, Bone remodeling in 1 representative animal treated with $50 \mathrm{~mW} / \mathrm{cm}^{2}$ where osteoclast cells (arrow) can be observed at 28 days (original magnification $40 \times$ ).

and were removed from further analyses. The rest of the animals had a good postoperative outcome without signs of respiratory insufficiency or flail chest.

\section{Histologic Findings}

Histologic features revealed that $26.3 \%$ of controls and $66 \%$ of PUS-treated animals were undergoing repair and remodeling $(P=.001)$ (Figure 1). The odds ratio for callus formation in animals treated was 5.4 (95\% CI, 2.1-13.9). During the first 10 days after surgery, only $41.6 \%$ of the animals treated with PUS and none of the control animals had calluses undergoing repair or remodeling. In contrast, when animals were treated for more than 10 days, $91.3 \%$ of the PUS group and $55.5 \%$ of controls were undergoing repair and remodeling $(P=.010)$. Repair or remodeling was observed in $73.3 \%$ of animals treated with $50 \mathrm{~mW} / \mathrm{cm}^{2}$, $68.8 \%$ with $100 \mathrm{~mW} / \mathrm{cm}^{2}$, and $56.3 \%$ with $250 \mathrm{~mW} / \mathrm{cm}^{2}$ (Figure 2, A). This was a significant improvement over controls $(P=.003$ for each group), but we observed no significant differences between the treatment groups $(P=.580)$. When we pooled the animals, $71 \%$ of animals treated with 50 and $100 \mathrm{~mW} / \mathrm{cm}^{2}$ were undergoing repair and remodeling, as compared with $56.3 \%$ of the animals in the $250 \mathrm{~mW} / \mathrm{cm}^{2}$ group $(P=.027)$. The odds ratio for callus formation was $7.7(95 \% \mathrm{CI}, 1.9-29.7)$ for $50 \mathrm{~mW} / \mathrm{cm}^{2}, 6.1(95 \% \mathrm{CI}$, 1.7-22.1) for $100 \mathrm{~mW} / \mathrm{cm}^{2}$, and $3.6(95 \% \mathrm{CI}, 1.0-12.2)$ for $250 \mathrm{~mW} / \mathrm{cm}^{2}$.

\section{Radiologic Findings}

An incipient or formed bone callus was observed in $26.3 \%$ of control animals as compared with $59.6 \%$ of treated animals $(P=.001)$. The odds ratio for callus formation in animals treated was 4.1 (95\% CI, 1.6-10.4). When we applied PUS for less than 10 days, only $29.1 \%$ of treated animals and none of the control group showed callus formation. In contrast, $91.3 \%$ of animals treated for more than 10 days and $55.5 \%$ of the control group had calluses $(P=.028)$. Callus or incipient bone formed in $73.3 \%$ of animals treated with 

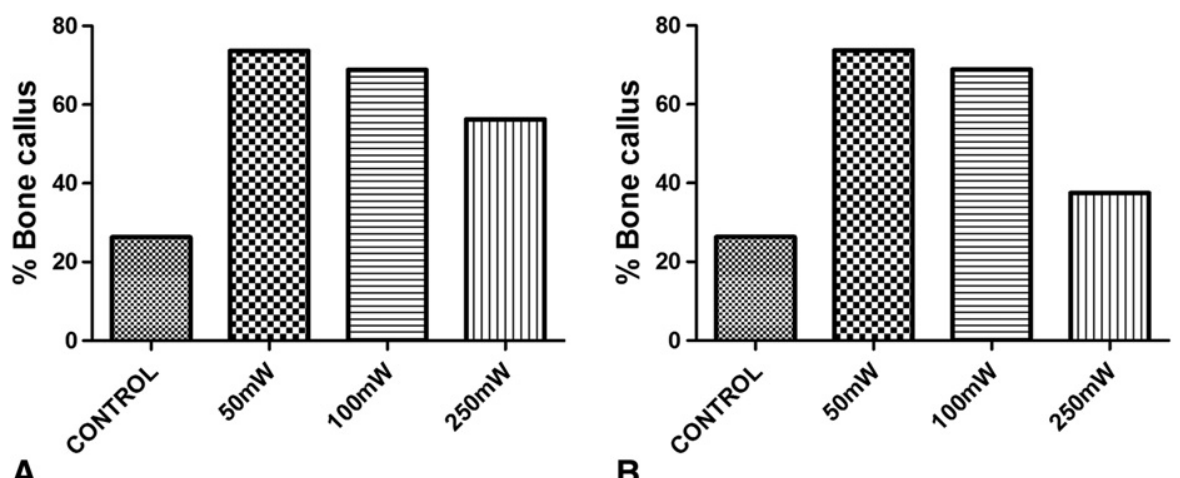

FIGURE 2. Histologic and radiologic bone consolidation with different doses of PUS. A, Histologic examination revealed that bone consolidation was higher in the pooled PUS treatment group than in the control group $(66 \%$ vs $26.3 \% ; P=.001)$. Consolidation at different doses of PUS was better than in the control groups $\left(P=.003\right.$ for each doses subgroup), although this improvement was lower with $250 \mathrm{~mW} / \mathrm{cm}^{2}$ than with lower doses $(50$ and 100 $\left.\mathrm{mW} / \mathrm{cm}^{2} \mathrm{I}_{\mathrm{SATA}} ; P=.027\right)$. B, Radiologic analysis revealed that incipient or formed bone callus was higher in the pooled PUS treatment group than in the control group $(59.6 \%$ vs $26.3 \% ; P=.001)$. Consolidation at different doses of PUS was better than in control group ( $P=.003$ for each doses subgroup), although this improvement was lower with $250 \mathrm{~mW} / \mathrm{cm}^{2}$ than with lower doses $\left(50\right.$ and $\left.100 \mathrm{~mW} / \mathrm{cm}^{2} \mathrm{I}_{\mathrm{SATA}} ; P=.027\right)$. PUS, Pulsed ultrasound; $I_{S A T A}$, spatial average-temporal average intensity.

$50 \mathrm{~mW} / \mathrm{cm}^{2}, 68.8 \%$ with $100 \mathrm{~mW} / \mathrm{cm}^{2}$, and $37.5 \%$ with 250 $\mathrm{mW} / \mathrm{cm}^{2}$ (Figure $2, B$ ). This was a significant radiologic improvement over controls ( $P=.003$ for each group), although we did not observe significant differences between the treatment groups $(P=.083)$. However, significant differences were found between the treatment groups when we evaluated the number of incipient and formed calluses for 50 and 100 $\mathrm{mW} / \mathrm{cm}^{2}$ combined $(71 \%)$ versus $250 \mathrm{~mW} / \mathrm{cm}^{2}(37.5 \%)$ $(P=.027)$. The odds ratio for callus formation was 7.7 $(95 \% \mathrm{CI}, 1.99-29.75)$ for $50 \mathrm{~mW} / \mathrm{cm}^{2}, 6.16(95 \% \mathrm{CI}$, $1.71-22.15)$ for $100 \mathrm{~mW} / \mathrm{cm}^{2}$, and $1.68(95 \% \mathrm{CI}, 0.48-$ 22.15) for $250 \mathrm{~mW} / \mathrm{cm}^{2}$.

\section{Gene Expression}

When we grouped all animals treated with PUS versus controls, we observed a significant increase in the expression of IGF1, SOCS2, and VEGFa (Table 1). When we evaluated the dose-dependent effects with regard to the time of treatment, we observed that $100 \mathrm{~mW} / \mathrm{cm}^{2}$ significantly increased $I G F 1$ expression at 10 and 28 days and SOCS2 at 28 days and decreased monocyte chemoattractant protein- 1 at 28 days. Similarly, $250 \mathrm{~mW} / \mathrm{cm}^{2}$ increased SOCS3 expression at 10 days and SOCS2, IGF1, and VEGFa at 10 and 28 days and decreased the expression of COL2al at 28 days. No significantly variations were observed with $50-\mathrm{mW} /$ $\mathrm{cm}^{2}$ PUS dose (Figure 3, $A$ and $B$ ).

\section{DISCUSSION}

The major finding of our study was that PUS was able to accelerate the consolidation of RF. Our study showed for the first time that the histologic and radiologic consolidation of $\mathrm{RF}$ is significantly improved over the natural healing process when animals are treated with PUS. Several experimental and clinical studies have previously examined the benefits of PUS for fracture repair of limbs, ${ }^{11}$ but no studies have reported the use of PUS in RF yet. Currently, there are no effective treatments to alter the functional complications of RF, which often last for several months and confer a high cost. As a result, some authors have suggested that

TABLE 1. Effects of total PUS treatment on transcriptional activity in the callus from rib fractures

\begin{tabular}{ccccc}
\hline Gene & Days of treatment & Control & PUS & $\boldsymbol{P}^{*}$ \\
\hline$I G F 1$ & 10 & $1.00 \pm 0.19$ & $1.47 \pm 0.37$ & .008 \\
& 28 & $1.00 \pm 0.14$ & $1.72 \pm 0.43$ & .003 \\
TGFb1 & 10 & $1.00 \pm 0.20$ & $1.07 \pm 0.23$ & .373 \\
& 28 & $1.00 \pm 0.51$ & $1.22 \pm 0.35$ & .322 \\
COL2al & 10 & $1.00 \pm 0.61$ & $0.99 \pm 0.85$ & .899 \\
& 28 & $1.00 \pm 1.13$ & $0.93 \pm 1.62$ & .563 \\
VEGFa & 10 & $1.01 \pm 0.26$ & $1.17 \pm 0.46$ & .703 \\
& 28 & $1.00 \pm 0.20$ & $1.62 \pm 0.69$ & .039 \\
MMP3 & 10 & $1.00(0.71-2.35)$ & $0.69(0.19-4.35)$ & .378 \\
& 28 & $1.00(0.56-5.41)$ & $0.78(0.19-3.28)$ & .635 \\
BMP2 & 10 & $1.00 \pm 0.19$ & $0.88 \pm 0.21$ & .227 \\
& 28 & $1.00 \pm 0.29$ & $1.15 \pm 0.32$ & .283 \\
SOCS2 & 10 & $1.00 \pm 0.67$ & $1.72 \pm 0.55$ & .019 \\
& 28 & $1.01 \pm 0.63$ & $1.98 \pm 0.55$ & .010 \\
SOCS3 & 10 & $1.00 \pm 0.95$ & $1.06 \pm 1.00$ & .799 \\
& 28 & $1.01 \pm 0.65$ & $1.70 \pm 0.93$ & .137 \\
MCP-1 & 10 & $1.00 \pm 0.91$ & $0.71 \pm 0.70$ & .181 \\
& 28 & $1.00 \pm 0.26$ & $0.99 \pm 0.51$ & .741 \\
\hline
\end{tabular}

Messenger RNA expression of genes normalized to polymerase II polypeptide A and presented as fold-change relative to control. Data are representative of 3 independent experiments and presented as mean $\pm \mathrm{SD} . M M P 3$ is expressed as median (minimum and maximum). PUS, Pulsed ultrasound; $I G F 1$, insulin-like growth factor $1 ; T G F b$, transforming growth factor beta; Col2al, collagen type II-alpha $1 ; V E G F a$, vascular endothelial growth factor alpha; $M M P 3$, matrix metallopeptidase $3 ; B M P 2$, morphogenetic bone protein 2; SOCS, suppressor of cytokine signaling; $M C P-1$, monocyte chemoattractant protein -1 . *The $P$ value estimates the differences between the control group and all PUS treatment groups and was computed using the Mann-Whitney $U$ test. 

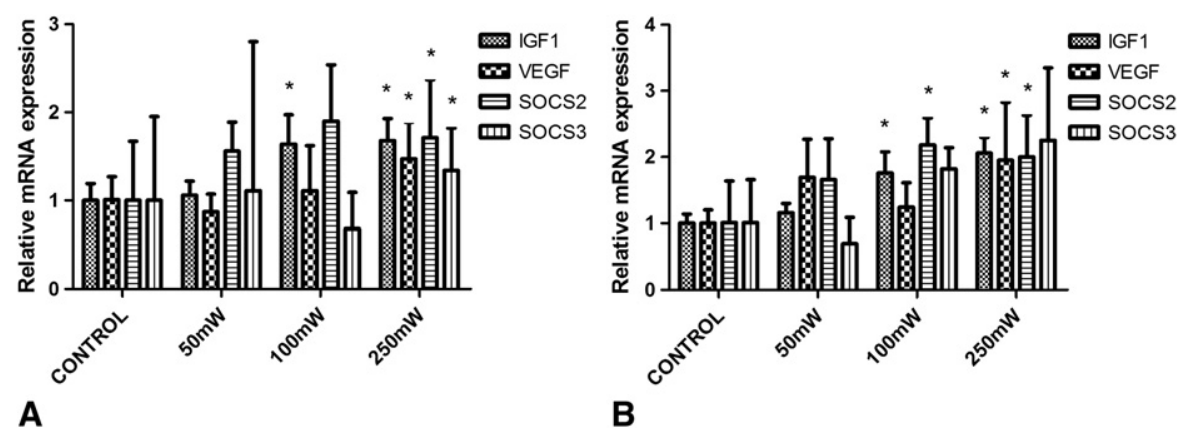

FIGURE 3. Messenger RNA expression of $I G F 1, V E G F$, SOCS2, and SOCS3. A, At 10 days, treatment with $100 \mathrm{~mW} / \mathrm{cm}^{2}$ increased IGF1 $(P=.004)$ and treatment with $250 \mathrm{~mW} / \mathrm{cm}^{2}$ increased SOCS3 $(P=.046)$, SOCS2 $(P=.045), I G F 1(P=.003)$, and VEGFa $(P=.032)$. No significant variations were observed with $50 \mathrm{~mW} / \mathrm{cm}^{2}$ after 10 days of treatment. Data are representative of 3 independent experiments and error bars indicate standard deviations from the mean. *Statistically significantly differences between control and PUS treatment groups. B, At 28 days, animals treated with $100 \mathrm{~mW} / \mathrm{cm}^{2} \mathrm{I}_{\mathrm{SATA}}$ group had significantly higher levels of IGF1 $(P=.006)$ and SOCS2 $(P=.018)$. Animals treated with $250 \mathrm{~mW} / \mathrm{cm}^{2}$ showed an increase of SOCS2 $(P=.045), I G F 1$ $(P=.006)$, and $\operatorname{VEGFa}(P=.028)$. No significant variations were observed with $50 \mathrm{~mW} / \mathrm{cm}^{2}$ after 28 days of treatment. Data are representative of 3 independent experiments. Error bars indicate standard deviations from the mean. *Statistically significantly differences between control and PUS treatment group. PUS, Pulsed ultrasound; $I_{S A T A}$, spatial average-temporal average intensity; $I G F 1$, insulin-like growth factor 1; VEGF, vascular endothelial growth factor; SOCS, suppressor of cytokine signaling.

developing effective treatment strategies for RF could reap large individual and social profits. ${ }^{13}$

It has been previously reported that longer duration of treatment is more effective for consolidation of fractures of inferior limbs in rats. ${ }^{14}$ Our study found that animals treated for more than 10 days had 5 times greater bone callus formation. This suggests that the rib repair induced by PUS requires a longer duration of treatment to accelerate the progression from the inflammatory phase to the repair phase. In our study, PUS was applied for just 3 minutes per day, which is much less time than the 15 minutes ${ }^{15}$ or 20 minutes ${ }^{14}$ often described for treatment of lower limb fractures in rats. This time does not appear to have limited the effectiveness of PUS on the RF, thus greatly shortening the treatment time and making it more tolerable and easier to apply. We observed that the consolidation decreased with the increasing intensity applied. In this sense, our data are consistent with those obtained for lower extremity fractures, in which the best results were obtained with $30 \mathrm{~mW} / \mathrm{cm}^{2}$. It is unlikely that this minor effect observed with $250 \mathrm{~mW} /$ $\mathrm{cm}^{2}$ on the RF consolidation is due to thermal damage, as it has been postulated with much higher intensities. ${ }^{16}$

The mechanism by which PUS proves beneficial is still largely unknown. There are evidences that PUS produces an increasing cellular activity. ${ }^{7}$ Several in vitro experiments have demonstrated that PUS treatment is capable of inducing the activation of several genes. A recent study ${ }^{17}$ with a crop of bone marrow cells from rats has been able to demonstrate that PUS increased the expression of genes involved in osteoblast proliferation and differentiation such as IGFI. Whether these effects occur in vivo, appear during prolonged PUS treatment, or relate to the therapeutic effect on the same fractures is still unclear. Our study confirmed that PUS induced significant changes in the expression of IGFI and $V E G F a$ and showed for the first time that PUS induced changes in SOCS2 and SOCS3 gene expression in early and late stages of the regeneration of RF. IGF is the most abundant growth factor in the bone matrix and a powerful stimulator of osteogenesis. ${ }^{18} I G F 1$ is produced by osteoblasts and stimulates bone formation by inducing cell proliferation, osteoblast differentiation, biosynthesis of collagen type I, osteoclastogenesis, and interaction osteoblast-osteoclasto. ${ }^{19}$ Generally $I G F I$ acts as a mitogenic factor that is widely distributed in the soft early stages in fracture callus repair. ${ }^{20}$ Its expression increases significantly after the third day, reaching a peak within 10 days, and then declines gradually until day 21 in femur fracture from rats. ${ }^{21}$ Furthermore, new blood vessels are known to form during the inflammatory stage of repair, and in vitro cell studies have demonstrated that PUS exposure increases the $V E G F$ expression in osteoblast. VEGF is the major regulator both in vitro and in vivo of angiogenesis in various pathophysiologic processes. This may stimulate angiogenesis, which is both a key component in early bone repair and a necessary precursor to endochondral ossification. ${ }^{11,17}$ We have observed a significant increase in the expression of $I G F I$ and $V E G F a$ in late stages of RF repair induced by PUS.

On the other hand, both SOCS2 and SOCS3 negatively regulate the signal transduction of several proinflamatory cytokines and IGF $1 .{ }^{22} \operatorname{SOCS} 2(-/)$ mice showed increased longitudinal skeletal growth associated with deregulated growth hormone/IGF1 signaling. Recently, it has been shown that physiologic levels of SOCS2 negatively regulate bone formation and endochondral growth. ${ }^{23}$ Accordingly, our results suggest that the increase of SOCS2 and SOCS3 expressions are associated with worst RF consolidation induced by PUS. In this sense, the highest dose increases the expression of IGF1,VEGF, SOCS2, and SOCS3, suggesting 
that they cause a tissue response directed to alleviate the situation of inflammation and tissue damage in response to PUS doses ${ }^{24}$ and decreased COL2al expression. In addition, it has been observed that doses of $30 \mathrm{~mW} / \mathrm{cm}^{2}$ promote deposition of calcium and the synthesis of collagen matrix mesh that supports bone mineralization, whereas exposure to higher intensities of ultrasound $120 \mathrm{~mW} / \mathrm{cm}^{2}$ produces a lower deposition of calcium. ${ }^{25} \mathrm{~A}$ deep molecular knowledge also helps to understand why the worst results in the consolidation of RF in our study were found in animals treated with higher PUS doses, and therefore higher doses must be avoided to treat RF, although we have not elucidated the mechanisms by which PUS is beneficial.

The main limitation encountered by our study was the difficulty to evaluate the possible analgesic effects of PUS in rats; therefore, we will have to wait for the clinical studies in humans to be able to determine this issue. An ongoing multicenter randomized clinical trial (supported by SEPAR, the Spanish Society of Neumology and Thoracic Surgery) is designed to evaluate further the effects of PUS in patients with rib fractures.

In conclusion, this is the first time that the beneficial effect of PUS on the consolidation of RF has been demonstrated. The healing rate of callus formation was greater than in controls and the ongoing clinical studies will confirm whether there is actual benefit.

We thank Yanira Brito Godoy, Pedro LLontop Santisteban, Dr Nicolás Díaz Chico, Dr Ricardo Chirino Godoy, and Dr José Carlos Rodríguez Pérez for their support.

\section{References}

1. Meredith JW, Hoth JJ. Thoracic trauma: when and how to intervene. Surg Clin North Am. 2007;87:95-118.

2. Sharma OP, Oswanski MF, Jolly S, Lauer SK, Dressel R, Stombaugh HA. Perils of rib fractures. Am Surg. 2008;74:310-4.

3. Bulger EM, Arneson MA, Mock CN, Jurkovich GJ. Rib fractures in the elderly. J Trauma. 2000;48:1040-6.

4. Flagel BT, Luchette FA, Reed RL, Esposito TJ, Davis KA, Santaniello JM, et al. Half-a-dozen ribs: the breakpoint for mortality. Surgery. 2005;138:717-23.

5. Karmakar MK, Ho AM. Acute pain management of patients with multiple fractured ribs. J Trauma. 2003;54:615-25.
6. Khandhar SJ, Johnson SB, Calhoon JH. Overview of thoracic trauma in the United States. Thorac Surg Clin. 2007;17:1-9.

7. Claes L, Wiilie B. The enhancement of bone regeneration by ultrasound. Prog Biophys Mol Biol. 2007;93:384-98.

8. Khan SN, Bostrom MP, Lane JM. Bone growth factors. Orthop Clin North Am. 2000;31:375-88.

9. Kristiansen TK, Ryaby JP, McCabe J, Frey JJ, Roe LR. Accelerated healing of distal radial fractures with the use of specific, low-intensity ultrasound. A multicenter, prospective, randomized, double-blind, placebo-controlled study. J Bone Joint Surg Am. 1997;79:961-73.

10. Leung KS, Lee WS, Tsui HF, Liu PP, Cheung WH. Complex tibial fracture outcomes following treatment with low-intensity pulsed ultrasound. Ultrasound Med Biol. 2004;30:389-5.

11. Khan Y, Laurencin CT. Fracture repair with ultrasound: clinical and cell-based evaluation. J Bone Joint Surg. 2008;90:138-44.

12. Rozen S, Skaletsky JH. Primer3 on the WWW for general users and for biologist programmers. Methods Mol Biol. 2000;132:365-86.

13. Kerr-Valentic MA, Arthur M, Mullins RJ. Rib fracture pain and disability: can we do better? J Trauma. 2003;54:1058-63.

14. Azuma Y, Ito M, Harada Y, Takagi H, Ohta T, Jingushi S. Low-intensity pulsed ultrasound accelerates rat femoral fracture healing by acting on the various cellular reactions in the fracture callus. J Bone Miner Res. 2001;16:671-80.

15. Wang SJ, Lewallen DG, Bolander ME, Chao EY, Ilstrup DM, Greenleaf JF. Low intensity ultrasound treatment increases strength in a rat femoral fracture model. J Orthop Res. 1994;12:40-7.

16. Fatar M, Stroick M, Griebe M, Alonso A, Hennerici MG, Daffertshofer M. Brain temperature during $340-\mathrm{kHz}$ pulsed ultrasound insonation: a safety study for sonothrombolysis. Stroke. 2006;37:1883-7.

17. Sena K, Leven RM, Mazhar K, Sumner DR, Virdi AS. Early gene response to low-intensity pulsed ultrasound in rat osteoblastic cells. Ultrasound Med Biol. 2005;31:703-8.

18. Lieberman JR, Daluiski A, Einhorn TA. The role of growth factors in the repair of bone. Biology and clinical applications. J Bone Joint Surg Am. 2002;84:1032-44.

19. Wang Y, Nishida S, Elalieh HZ, Long RK, Halloran BP, Bikle DD. Role of IGF-I signaling in regulating osteoclastogenesis. J Bone Miner Res. 2006;21:1350-8.

20. Franceschi RT. Biological approaches to bone regeneration by gene therapy. J Dent Res. 2005;84:1093-103.

21. Hadjiargyrou M, Lombardo F, Zhao S, Ahrens W, Joo J, Ahn H, et al. Transcriptional profiling of bone regeneration. Insight into the molecular complexity of wound repair. J Biol Chem. 2002;277:30177-82.

22. Yoshimura A, Naka T, Kubo M. SOCS proteins, cytokine signalling and immune regulation. Nature. 2007;7:1-12.

23. Macrae VE, Horvat S, Pells SC, Dale H, Collinson RS, Pitsillides AA, et al. Increased bone mass, altered trabecular architecture and modified growth plate organization in the growing skeleton of SOCS2 deficient mice. J Cell Physiol. 2009;218:276-84.

24. Feril LB, Kondo T. Biological effects of low intensity ultrasound: the mechanism involved, and its implications on therapy and on biosafety of ultrasound. J Radiat Res (Tokyo). 2004;45:479-89.

25. Saito M, Soshi S, Tanaka T, Fujii K. Intensity-related differences in collagen posttranslational modification in MC3T3-E1 osteoblasts after exposure to low- and high-intensity pulsed ultrasound. Bone. 2004;35:644-55. 